ARTICLE

Received 23 Feb 2015 | Accepted 4 May 2015 | Published 10 Jun 2015

DOI: $10.1038 /$ ncomms8387

OPEN

\title{
Architecture of TFIIIC and its role in RNA polymerase III pre-initiation complex assembly
}

\author{
Gary Male ${ }^{1}$, Alexander von Appen ${ }^{1}$, Sebastian Glatt ${ }^{1}$, Nicholas M.I. Taylor ${ }^{1} \uparrow$, Michele Cristovao ${ }^{1}$, Helga Groetsch ${ }^{1}$ \\ Martin Beck ${ }^{1} \&$ Christoph W. Müller ${ }^{1}$
}

In eukaryotes, RNA Polymerase III (Pol III) is specifically responsible for transcribing genes encoding tRNAs and other short non-coding RNAs. The recruitment of Pol III to tRNAencoding genes requires the transcription factors (TF) IIIB and IIIC. TFIIIC has been described as a conserved, multi-subunit protein complex composed of two subcomplexes, called $\tau \mathrm{A}$ and $\tau B$. How these two subcomplexes are linked and how their interaction affects the formation of the Pol III pre-initiation complex (PIC) is poorly understood. Here we use chemical crosslinking mass spectrometry and determine the molecular architecture of TFIIIC. We further report the crystal structure of the essential TPR array from $\tau A$ subunit $\tau 131$ and characterize its interaction with a central region of $\tau B$ subunit $\tau 138$. The identified $\tau 131-\tau 138$ interacting region is essential in vivo and overlaps with TFIIIB-binding sites, revealing a crucial interaction platform for the regulation of tRNA transcription initiation.

\footnotetext{
${ }^{1}$ European Molecular Biology Laboratory (EMBL), Structural and Computational Biology Unit, Meyerhofstrasse 1, Heidelberg 69117, Germany. $\dagger$ Present address: Ecole Polytechnique Federale de Lausanne, BSP 416 (Cubotron UNIL), Route de la Sorge, CH-1015 Lausanne, Switzerland. Correspondence and requests for materials should be addressed to C.W.M. (email: cmueller@embl.de).
} 
E ukaryotes require the multi-subunit Pol III to transcribe genes encoding small, non-coding RNAs including tRNAs ${ }^{1}$. Recruitment of Pol III to these genes requires the essential transcription factors TFIIIB and TFIIIC ${ }^{2}$. TFIIIB is assembled from three subunits, namely Brf1, Bdp1 and $\mathrm{TBP}^{3}$. TFIIIC harbours six proteins in yeast, and has a molecular weight of approximately $500 \mathrm{kDa}$ (ref. 4). The six subunits of yeast TFIIIC are partitioned into two DNA-binding subcomplexes ( $\tau \mathrm{A}$ and $\tau \mathrm{B}$ ). Each subcomplex binds to its respective, highly conserved promoter sequence, named 'A box' and 'B box ${ }^{5,6}$. The binding of these intragenic promoters by TFIIIC permits the assembly of TFIIIB, upstream of the transcription start site, which subsequently leads to the recruitment of Pol III, the formation of a PIC and finally to transcription.

Both in vitro and in vivo studies have demonstrated that TFIIIC is a highly flexible protein complex that can accommodate the varying lengths of DNA sequence between the $\mathrm{A}$ and $\mathrm{B}$ boxes of different tRNA genes ${ }^{7,8}$. It is hypothesized that a flexible linker between the $\tau \mathrm{A}$ and $\tau \mathrm{B}$ subcomplexes allows TFIIIC to fulfil these requirements ${ }^{9,10}$. In yeast, $\tau \mathrm{A}$ is composed of $\tau 131$ (Tfс4), $\tau 95$ (Tfc1) and $\tau 55$ (Tfc7) and $\tau \mathrm{B}$ of $\tau 138$ (Tfc3), $\tau 91$ (Tfc6) and $\tau 60$ $(\mathrm{Tfc} 8)^{5}$. All six subunits are essential in vivo ${ }^{3}$. In human, orthologues of all six yeast subunits have been identified, with sequence similarity highest amongst the $\tau$ A subunits ${ }^{11}$.

While a significant amount of high-resolution structural and functional information exists for some individual TFIIIC subunits $^{12-14}$, a detailed understanding of how the $\tau \mathrm{A}$ and $\tau \mathrm{B}$ subcomplexes are connected and which subunits could act as a flexible linker remains elusive (Fig. 1a). $\tau 95$ of $\tau$ A has been proposed as a linker, based on co-immunoprecipitation experiments that show binding of this subunit to the $\tau \mathrm{B}$ subunits $\tau 138$ and $\tau 91$ (ref. 15). A genetic study suggests that the two largest TFIIIC subunits, $\tau 138$ and $\tau 131$, are responsible for connecting $\tau \mathrm{A}$ and $\tau \mathrm{B}^{16}$. The $\tau 138$ subunit is currently the least well-characterized subunit of TFIIIC. Photochemical crosslinking studies support the view that $\tau 138$ recognizes the $\mathrm{B}$ box promoter of tRNA genes ${ }^{17}$, as does the mutation of a highly conserved glycine residue (G349E) within $\tau 138$ that dramatically reduces the affinity of TFIIIC for tDNA ${ }^{18}$. Interestingly, this mutation can be suppressed by point mutations within the $\tau 131$ subunit, suggesting a direct physical interplay between these two subunits ${ }^{16} . \tau 131$ shows the highest sequence conservation of the TFIIIC subunits and is predicted to contain several tetra-trico peptide repeats (TPRs) ${ }^{19}$. TPRs typically contain 34 amino acids arranged into two antiparallel alpha-helices, with proteins often containing several of these motifs in an array ${ }^{20}$. This arrangement can lead to an extended, right-handed super-helical structure, which can provide extended binding surfaces for other subunits within a protein assembly ${ }^{21}$. $\tau 131$ fulfills this role within TFIIIC, binding to subunits Brf1 and Bdp1 in a stepwise mechanism to help assemble TFIIIB at tRNA genes, utilizing overlapping sites on an amino-terminal (N-terminal) 'TPR array'19,22-28. It has been further hypothesized that the relative positioning of the TPRs and the extended $\tau 131 \mathrm{~N}$ terminus mask binding sites within the TPR array, leading to an auto-inhibited $\tau 131$ state that must be relieved to allow Brf1 and Bdp1 binding ${ }^{27,28}$. These rate-limiting steps in Pol III PIC formation may be overcome, at least in part, by flexibility within $\tau 131$. Indeed, previous studies suggest conformational changes take place within $\tau 131$ on the binding of Brf1 and Bdp1 (refs 25,29). Finally, $\tau 131$ has also been shown to bind Pol III subunits Rpc53 and $\mathrm{ABC} 10 \alpha^{30,31}$. Molecular details of the interactions of $\tau 131$ with $\tau \mathrm{A}$ or $\tau \mathrm{B}$ subunits have not been reported, although in humans it has been shown that the $\tau 131$ orthologue (TFIIIC102) interacts with the $\tau 95$ orthologue (TFIIIC63), again requiring the conserved TPR array $^{32}$.
We set out to characterize the molecular architecture of TFIIIC and identify the $\tau \mathrm{A}-\tau \mathrm{B}$ linker region within TFIIIC using a combination of structural and biochemical approaches. Our findings implicate a central role for the TPR array of $\tau 131$ in linking $\tau \mathrm{A}, \tau \mathrm{B}$ and TFIIIB to regulate the formation of the Pol III PIC.

\section{Results}

The $\tau 131$ TPR array crosslinks to a central region of $\tau 138$. As a first step in identifying the link between $\tau \mathrm{A}$ and $\tau \mathrm{B}$ subcomplexes, we performed a chemical crosslinking mass spectrometry (XL-MS) approach on purified, endogenous TFIIIC from S. cerevisiae. The protein complex was purified from a yeast strain, carrying a tandem affinity purification (TAP) tag on the $\tau 60$ subunit. Pure and stoichiometric TFIIIC could be obtained as assessed by size-exclusion chromatography and SDS-polyacrylamide gel electrophoresis (SDS-PAGE; Fig. 1b). The complex was shown to be functional by its ability to bind a double-stranded oligonucleotide containing $\mathrm{A}$ and $\mathrm{B}$ box sequences by electrophoretic mobility shift assay (EMSA; Fig. 1c). Using XL-MS, we identified 33 unique intersubunit and 89 unique intrasubunit crosslinks with high confidence at a linear discriminant (ld) score of $>25$ (Fig. 1d and Supplementary Tables 1 and 2). Twelve of the intersubunit crosslinks bridge $\tau 131$ with $\tau 138$. The majority of these links connect the N-terminal TPR array of $\tau 131$ to an unstructured, central region of $\tau 138$. With the exception of three additional crosslinks identified between the $\tau 95$ subunit and $\tau 138$, these links represent the major $\tau \mathrm{A}-\tau \mathrm{B}$ connection revealed by our approach. Our XL-MS analyses also provide additional insights into the overall architecture of TFIIIC. Within the $\tau \mathrm{A}$ subcomplex, six crosslinks connect the dimerization domains of $\tau 95$ and $\tau 55$, consistent with our previously reported crystal structure $^{14}$. In addition, we identified four crosslinks between the $\tau 95$ subunit and predicted TPRs at the C terminus of $\tau 131$. Within the $\tau \mathrm{B}$ subcomplex, there are surprisingly few crosslinks observed between the $\tau 60$ and $\tau 91$ subunits, despite the large interface between them ${ }^{12}$. Xlink Analyzer ${ }^{33}$ shows that some lysine residues are entirely buried in the $\tau 60-\tau 91$ interface and are therefore not accessible to the crosslinker. We also observe lysine residues surrounding this interface that are not detected by our XL-MS analysis, probably because they are buried in interfaces with other TFIIIC subunits. Four crosslinks connect $\tau 91$ with a disordered region between two predicted winged helix domains of $\tau 138$, which is consistent with the proposal of a co-operative role between $\tau 91$ and $\tau 138$ for B box binding ${ }^{34}$.

We went further in our analysis of the entire TFIIIC complex by performing XL-MS on DNA-bound TFIIIC, to determine whether the complex undergoes large conformational changes (Supplementary Tables 3 and 4). In general, we observed no fundamental changes in the intersubunit interaction network on tDNA binding (compare Fig. 1d and Supplementary Fig. 1). In particular, the bridge between the $\tau 131$ and $\tau 138$ subunits is maintained, indicating the importance of this link for the integrity and stability of TFIIIC when bound to DNA. Interestingly, the four crosslinks connecting the $\tau 91$ and $\tau 138$ subunits are not detected when TFIIIC is bound to DNA, suggesting a local change in conformation that prevents these previously crosslinked residues from accessing the crosslinker. It is tempting to speculate that this region is therefore involved in direct binding of TFIIIC to DNA.

Structure of the $\tau 131$ TPR array. $\tau 131$ and $\tau 138$ are the largest subunits of the $\tau \mathrm{A}$ and $\tau \mathrm{B}$ subcomplexes, respectively ${ }^{5}$. $\tau 131$ was predicted to contain a highly conserved TPR array architecture at the $\mathrm{N}$ terminus with additional conserved TPRs predicted at the 
a
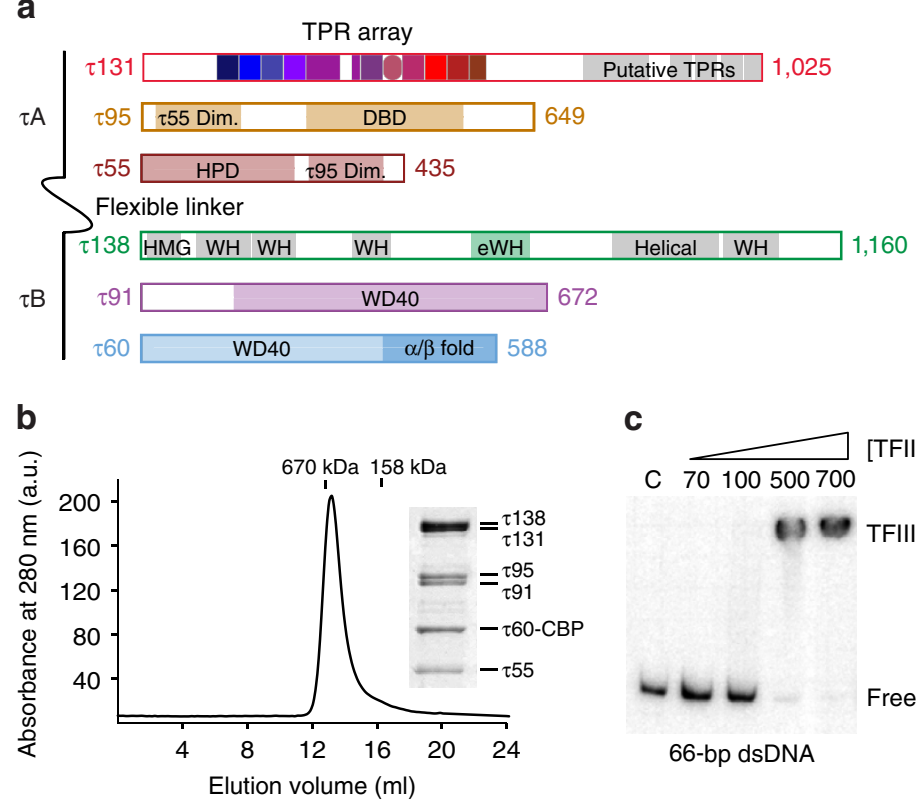

C

$\overparen{70100500700}[$ TFIIIC] $\mathrm{nM}$

d

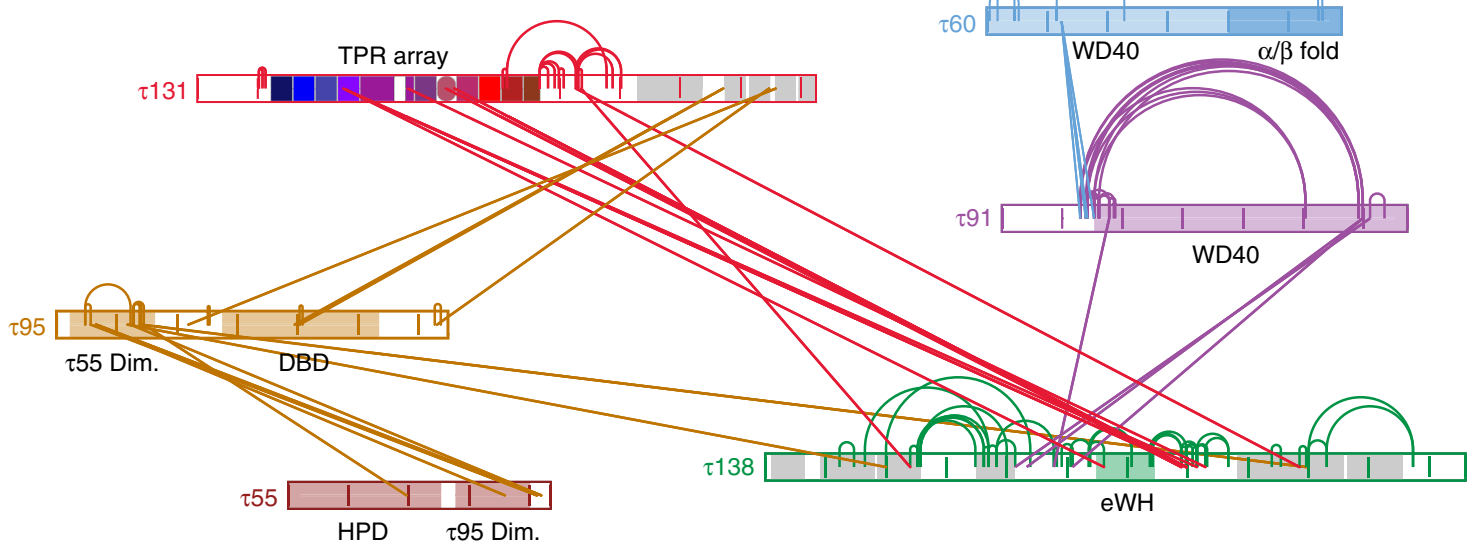

Figure 1 | XL-MS of purified, endogenous TFIIIC reveals a link between $\tau$ A and $\tau \mathbf{B}$. (a) Schematic representation of the six subunits of S. cerevisiae TFIIIC. The amino-acid lengths of the subunits are labelled at the $\mathrm{C}$ terminus. Domains of which crystal structures are available are highlighted. $\tau 55$ and $\tau 95$ Dim. $=\tau 55$ and $\tau 95$ dimerization domains; DBD, DNA-binding domain; HPD, histidine phosphatase domain. The tetra-trico peptide (TPR) array of $\tau 131$ and the eWH domain of $\tau 138$ are included, see text for details. Additional predicted structural regions of $\tau 131$ and $\tau 138$ are highlighted in grey. HMG, high mobility group box domain; $\mathrm{WH}=$ winged helix. (b) Analytical size-exclusion chromatography profile of TFIIIC using a Superose $610 / 300$ column (GE Healthcare). Known molecular weight standards at 670 and $158 \mathrm{kDa}$ are indicated. Inset, Coomassie-stained SDS-PAGE gel of an elution peak fraction. (c) EMSA experiment of TFIIIC bound to a double-stranded (ds) 66 base-pair (bp) tDNA Glu oligonucleotide. C, control (no TFIIIC added). (d) Crosslinking map of TFIIIC. TFIIIC subunits are represented as in a with internal vertical lines representing 100 amino-acid markers. Intra crosslinks are depicted by arcs that connect residues within the same subunit. Inter crosslinks are depicted by lines which connect residues within different subunits. Image produced using $x \mathrm{NNET}^{50}$.

C terminus ${ }^{19}$ (Fig. 2a). Given that our crosslinking data implicated the TPR array of $\tau 131$ in connecting $\tau A$ and $\tau B$, and the reported importance of this region for TFIIIB assembly, we determined the crystal structure of the TPR array (residues 123-566) in two different space groups $\left(\mathrm{P}_{2}\right.$ and $\left.\mathrm{P} 4_{3}\right)$. Starting from a construct expressing residues $1-580$ of $\tau 131$, we identified a stable fragment by limited proteolysis and mass spectrometry corresponding to residues 123-566. We expressed this truncated S. cerevisiae $\tau 131$ (123-566) protein in E. coli, purified it using affinity and size-exclusion chromatography and subsequently carried out crystallization trials. We were able to collect diffraction data up to $3.15 \AA\left(\mathrm{P}_{2}\right)$ and $3.4 \AA\left(\mathrm{P} 4_{3}\right)$ resolution. The lower-resolution structure was solved by multiple isomorphous replacement with anomalous signal (MIRAS), using anomalous signal from crystals prepared with selenomethionine substituted proteins or native crystals soaked with p-chloromercuribenzensulfonic acid to final $R_{\text {work }} / R_{\text {free }}$ values of $20.9 \% / 24.5 \%$ The higher-resolution structure was solved using selenomethionine substituted protein in single anomalous dispersion (SAD) experiments and subsequently refined to $R_{\text {work }} / R_{\text {free }}$ values of $25.0 \% / 28.7 \%$. The combination of selenomethionine and mercury $(\mathrm{Hg})$ markers aided the building of the structures. (Table 1, Supplementary Figs 2 and $3 a$ and Supplementary Table 5).

The structure reveals the presence of 10 TPR repeats, while only nine TPR repeats had been predicted. Rather than forming an extended super-helix, the TPR array is instead separated into two 'arms' by a central region that contains an extended helix and 
a

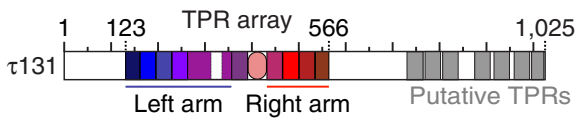

b
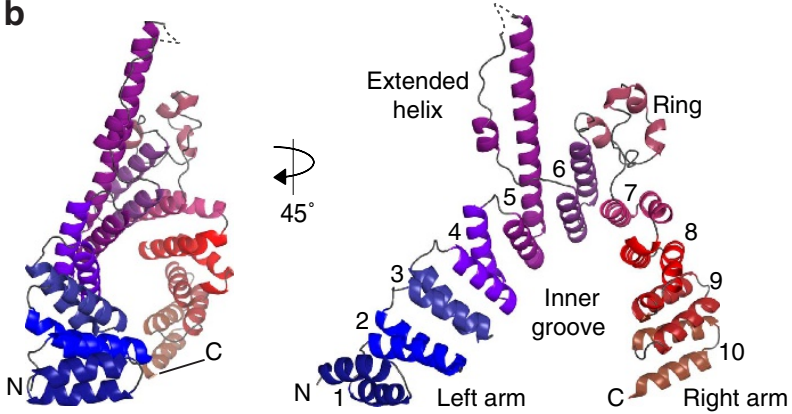

C

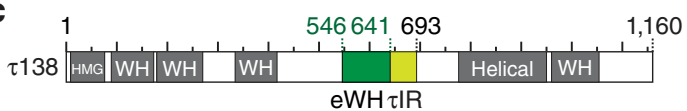

d
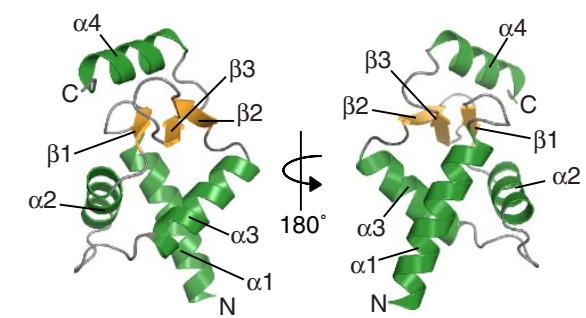

$\mathrm{N}-$

B1

$\beta 2 \beta 3$

641

Figure 2 | Crystal structures of the TPR array of $\tau 131$ and the central extended winged helix (eWH) domain of $\tau$ 138. (a) Schematic domain architecture of $\tau 131$. The TPR array is highlighted and coloured according to the solved crystal structure in $\mathbf{b}$. Putative TPRs are indicated in grey.

(b) Crystal structure of the $\tau 131$ (123-566) TPR array in ribbon

representation. Two views are displayed, related by a $45^{\circ}$ rotation. A dashed line indicates a region of the electron density where no residues could be built with confidence (residues 317-336). TPRs are numbered 1-10.

(c) Schematic domain architecture of $\tau 138$. Predicted winged helix $(\mathrm{WH})$ domains, the high mobility group (HMG)-box domain and the helical region are shaded in grey. The central eWH domain is shaded in dark green. The $\tau \mid \mathrm{R}$ is shaded in light green (see text for details). (d) Crystal structure of the $\tau 138$ (546-641) eWH domain in ribbon representation. Two views are displayed, related by a $180^{\circ}$ rotation. A schematic of the arrangement of $\alpha$-helices and $\beta$-strands is displayed underneath the structure.

a disordered coil we call 'ring' domain (Fig. 2b). This insertion causes a bend in the TPR array, positioning the TPRs of the right arm closer to the TPRs of the left arm. This feature generates a potential binding pocket we call the 'inner groove'. $\tau 131$ is postulated as being highly flexible, consistent with its role in binding to multiple ligands at different stages in Pol III PIC assembly. A superimposition of the two crystal forms and an analysis of temperature factors reveal flexibility at TPRs 1-3, 8-10 and residues at the extremes of the extended helix (Supplementary Fig. 3b). Despite crystal contacts in these regions, the accommodation of the TPR array in these arrangements in two different crystal forms suggests that the conformational changes are indeed permitted within the TPR array.

Characterization of the $\tau 131-\tau 138$ interaction region. We next wanted to characterize further the central region of $\tau 138$
(546-693) and understand which regions were crucial for interaction with the TPR array. We hypothesized that the inner groove of the TPR array may accommodate the predicted central winged helix domain of $\tau 138$ (Fig. 2c). Surface analysis of the TPR array shows that the inner groove is lined with patches of conserved, often acidic surface residues that may bind to the predicted basic surface residues of $\tau 138$ (Supplementary Figs 3c,d,e and 4). We first solved the structure of the central domain of $\tau 138$ (546-641) using the sulphur-SAD technique (Table 1, Supplementary Table 5, Supplementary Fig. 5a). The $1.4 \AA$ crystal structure $\left(R_{\text {work }} / R_{\text {free }}\right.$ of $\left.17.5 \% / 19.8 \%\right)$ of this domain reveals a canonical winged helix domain that contains an additional C-terminal helix (Fig. 2d). This first structurally characterized part of $\tau 138$ thus represents an 'extended' winged helix $(\mathrm{eWH})$ domain $^{35}$. The eWH domain is moderately well conserved from yeast to human (Supplementary Fig. 5b,c,d), and contains basic patches that could also suggest a role in binding nucleic acids (Supplementary Fig. 5e). However, we only detect very weak unspecific binding of the eWH domain to single and double-stranded DNA (Supplementary Fig. 5f). Isothermal titration calorimetry (ITC) did not detect an interaction between the eWH and the TPR array (Supplementary Fig. 6a). However, when we tested a construct that contained the eWH domain with additional residues at the C terminus (546-693), we observed a $K_{\mathrm{d}}$ for the interaction with the TPR array of $\sim 100 \mathrm{~nm}$ (Fig. 3a). A similar high-affinity interaction $(\sim 80 \mathrm{~nm})$ could be measured with only the unstructured region of $\tau 138$ (641-693) (Fig. 3b). Removal of residues $682-693$ lowered the $K_{\mathrm{d}}$ of the interaction to $\sim 2.6 \mu \mathrm{M}$ (Supplementary Fig. 6b), yet the region 641-681 was still essential to ensure the high-affinity interaction as tested peptides of 681-693 did not interact with the TPR array by ITC (data not shown). We thus concluded that the region $641-693$ of $\tau 138$, hereafter referred to as ' $\tau 131$-Interaction Region ( $\tau$ IR)', is necessary and sufficient to bind the TPR array of $\tau 131$.

To assess the in vivo importance of our in vitro results, we disrupted the chromosomal copy of $\tau 138$ in yeast and introduced a plasmid carrying wild-type $\tau 138$ and the URA3 gene under the control of the endogenous promoter, to maintain cell viability. This plasmid was then shuffled with a second plasmid carrying either the wild-type $\tau 138$ or a deletion mutant of $\tau 138$. We observed growth for cells transformed with the $\tau 138$ wild-type plasmid on FOA medium, but cells carrying the $\tau 138 \Delta \mathrm{eWH}-\tau \mathrm{IR}$ or the $\Delta \tau$ IR plasmid did not survive (Fig. 3c). This is a clear indication that these deletion mutants cannot complement the loss of wild-type $\tau 138$. Removal of residues $681-693$ leads to an intermediate phenotype of reduced yeast growth (Supplementary Fig. 6c). Interestingly, the removal of just the eWH domain is also lethal to yeast (Fig. 3c), indicating that despite it being dispensable for the interaction with $\tau 131$ in vitro, it is still essential for proper TFIIIC function in vivo.

$\tau 138$ and Bdp1 binding is affected by mutations in TPR 8. Our structure of the $\tau 131$ TPR array allows us, for the first time, to map and analyse mutations that have been previously described (Fig. 4a). In detail, mutations that increase Pol III transcription cluster mostly on TPR 2 (ref. 26), those that decrease Pol III transcription spread over TPRs 8-10 (ref. 22) and those that rescue a $\tau 138$ temperature-sensitive mutation, map mostly to TPRs 7-8 (ref. 16). Having mapped the critical $\tau 131$ interaction region of $\tau 138$ to $\sim 50$ amino acids, we next questioned where the $\tau$ IR binds on the TPR array. Despite extensive efforts, we were unable to obtain structural information of the $\tau 131-\tau$ IR complex although both polypeptides form a stable complex during size-exclusion chromatography. Instead, we analysed previously described mutations together with the surface conservation of the 
Table 1 | Data collection and refinement statistics.

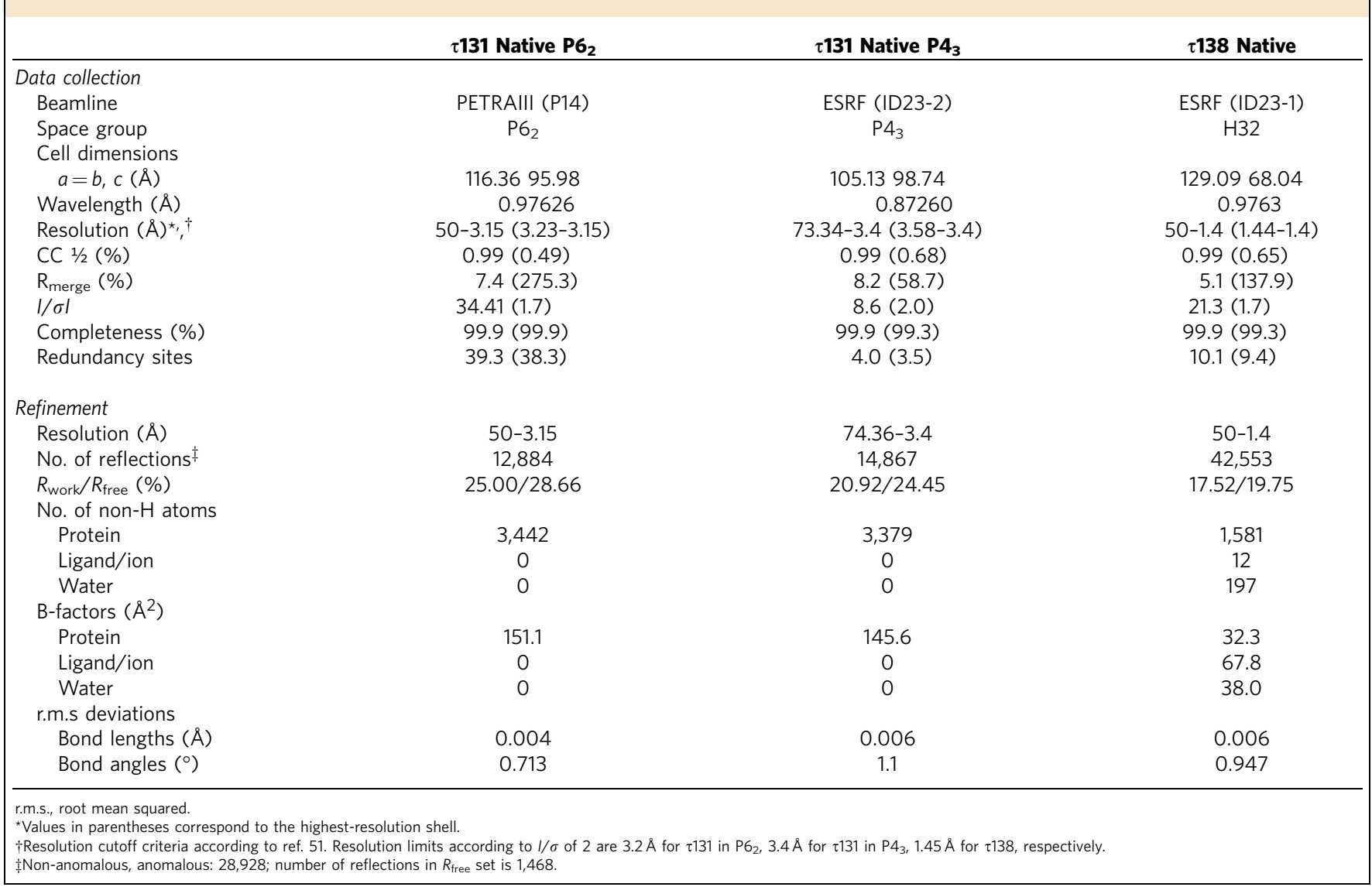

TPR array (Supplementary Fig. 3e), to predict where the $\tau$ IR may bind. We expressed and purified five $\tau 131$ point mutants that all cluster on TPR 8 (Fig. 4b). With the exception of residue L469, all of these residues are acidic and surface exposed (Fig. 4c). The purified mutants all eluted at the same volume from a sizeexclusion column when compared with the wild type, indicating that the mutations did not cause a destabilization of the proteins (Supplementary Fig. 7a). Using ITC, we determined binding affinities of the mutant $\tau 131$ TPR arrays to the $\tau$ IR and the eWH- $\tau$ IR (Fig. 4d and Supplementary Fig. 7b). No binding of the mutants D468K and L469K to the $\tau 138$ proteins could be detected by ITC. Mutants E472K and E498K showed much weaker binding, while the E497K mutant showed no significant decrease in binding affinity to the $\tau 138$ proteins. These findings are consistent with results from GST pull-down assays (Fig. 4e and Supplementary Fig. 7c). We note that the L469K mutation likely causes a steric clash in the packing of TPR 8 with TPR 7 rather than abolishing a site-specific contact (Fig. 4c). Strikingly, the mutation of residues D468 and L469 has been previously implicated in a loss of binding to TFIIIB subunit Bdp1 (refs 16,22). We purified full-length, recombinant Bdp1 and performed GST pull-down assays. Bdp1 binding to the TPR array of $\tau 131$ is strongly reduced in the $\mathrm{D} 468 \mathrm{~K}$ or $\mathrm{L} 469 \mathrm{~K}$ mutants (Fig. $4 \mathrm{f}$ ). To our knowledge, this is the first time that a loss of interaction by both of these mutations has been probed directly using purified proteins. These results suggest that the binding hotspot for $\tau 138$ thus overlaps with that of a binding site for Bdp1.

Brf1-TBP binds a distinct site from that of $\tau 138$ and Bdp1. Previous studies have proposed that $\tau 131$ exists in an autoinhibited form before assembly of TFIIIB ${ }^{27}$. This auto-inhibition, possibly by the masking of the $\tau 131$ TPR array by the extended $N$ terminus, may be relieved before or on recruitment of the Brf1 subunit of TFIIIB, paving the way for TBP and Bdp1 recruitment ${ }^{28}$. To further our understanding of the overlap between TFIIIB and $\tau 138$ binding, we purified a form of $\tau 131$ that contained the extended $\mathrm{N}$ terminus $(1-580)^{25}$ as a GST fusion for interaction studies. As recombinant Brfl is highly unstable, we were unable to produce sufficient quantities of pure, recombinant Brf1 protein for our studies. We therefore turned to a Brf1-TBP fusion protein that has been previously described and shown to functionally replace Brfl both in vitro and in vivo ${ }^{36}$.

We tested the binding of $\tau 138$ (eWH- $\tau$ IR) and Bdp1 to $\tau 131$ (1-580) (Fig. 5a). Both proteins bind stoichiometrically to this longer $\tau 131$ protein, showing that the extended $\mathrm{N}$ terminus of $\tau 131$ does not inhibit binding. We did not observe binding of Brf1-TBP to $\tau 131$ (123-566) under our experimental conditions (data not shown); however, we did detect an interaction using $\tau 131$ (1-580; Fig. 5a). The interaction was also observed with the D468K and the L469K mutants of $\tau 131$ (1-580) (Fig. 5a). These results indicate that the extended $\mathrm{N}$ terminus of $\tau 131$ is required for high-affinity binding to Brf1-TBP, and that the principal binding site on $\tau 131$ is separate from the hotspot of $\tau 138$ and Bdp1. The purified Brf1-TBP contained degradation products of the fusion protein corresponding to truncations lacking the $\mathrm{N}$ terminus of Brf1, as confirmed by MS (Supplementary Fig. 8). We observed that these degradation products were not pulled down by $\tau 131$ (1-580; Fig. 5a), suggesting that the $\mathrm{N}$ terminus of Brf1, which includes the TFIIB-like cyclin repeats, is required for the interaction.

To conclusively determine if the $\tau 138$ binding site overlaps with Bdp1 and/or Brf1-TBP, we next used the GST- $\tau 131$ (1-580) 
a

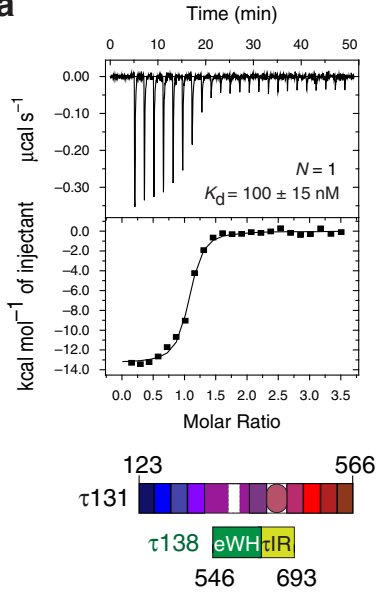

C

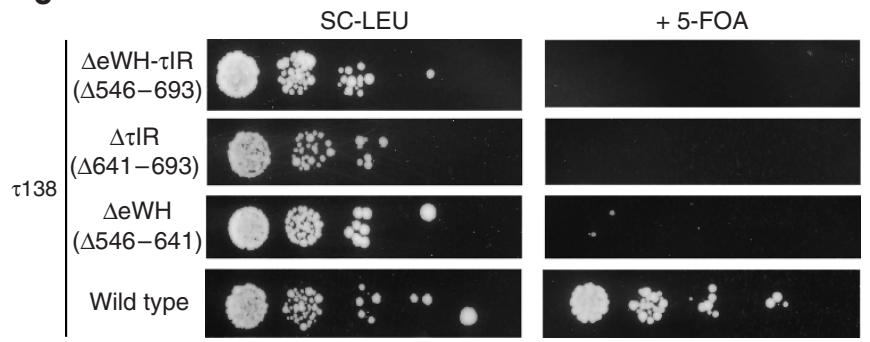

Figure 3 | The $\tau 131$ TPR array interacts with high affinity to a central region of $\tau$ 138. ITC measurement using purified (a) $\tau 138$ (546-693) and $\tau 131$ (123-566); (b) $\tau 138$ (641-693) and $\tau 131$ (123-566); Calculated $K_{d}$ values and stoichiometry $(N)$ are indicated. $15 \mu \mathrm{m}$ of $\tau 138$ was used in the cell and $150 \mu \mathrm{M} \tau 131$ was used in the syringe in each case. (c) Viability of $\tau 138$ deletion mutants in vivo determined by the spot assay. A yeast strain carrying the plasmid pOL49 was transformed with the plasmids pRS415 $\Delta$ CEN $\tau 138$ and pRS415 $\Delta$ CEN $\Delta 546-693$ or $\Delta 641-693$ or $\Delta 546-641$ and plated on SC-URA-LEU medium. Serial 10-fold dilutions of all strains were spotted on SC-LEU and 5-fluoroorotic acid medium and were incubated for $72 \mathrm{~h}$ at $30^{\circ} \mathrm{C}(n=3)$.

and incubated it with $\tau 138$ (eWH- $\tau \mathrm{IR}$ ), before titrating in increasing amounts of Bdp1 or Brf1-TBP. We observed a direct competition between $\tau 138$ and Bdp1 for binding to $\tau 131$ (Fig. $5 \mathrm{~b}$ ). These results were also replicated when using the shorter, TPR array of $\tau 131$ (data not shown). In contrast, even a $\sim 17$-fold molar excess of Brf1-TBP could not compete out the $\tau 138-\tau 131$ interaction (Fig. 5 c). Instead, both Brf1-TBP and $\tau 138$ can bind simultaneously to $\tau 131$, indicating that their binding sites are distinct.

\section{Discussion}

On the basis of limited proteolysis and low-resolution scanning transmission light microscopy, TFIIIC has long been described as a 'dumb-bell' shaped molecule consisting of two DNA-binding subcomplexes called $\tau \mathrm{A}$ and $\tau \mathrm{B}$ connected by a flexible linker ${ }^{9,10}$. More detailed information about the overall architecture of TFIIIC has been lacking. By combining structural information of individual subunits with our crosslinking data sets, we are able to provide a first model of the overall TFIIIC architecture (Fig. 6a). Our XL-MS data, combined with in vitro and in vivo mapping, indicate that the $\tau$ IR establishes the main link between $\tau \mathrm{A}$ and $\tau \mathrm{B}$, while the adjacent disordered regions on both sides of the eWH domain in $\tau 138$ presumably provide the necessary flexibility for binding variously spaced A box and B box promoters (Figs $2 \mathrm{c}$ and $6 a)$. The N-terminal TPR array of $\tau 131$ provides a docking platform for the $\tau \mathrm{IR}$, while C-terminal TPRs interact with the $\tau 95$ subunit of $\tau \mathrm{A}$. Thus, $\tau 131$ is crucial for linking the $\tau \mathrm{A}$ and $\tau \mathrm{B}$ subunits. Subunits $\tau 55$ and $\tau 95$, the two other subunits of the $\tau \mathrm{A}$ subcomplex, share an unexpected structural similarity with the general transcription factor TFIIF $^{14}$. Subunits $\tau 55$ and $\tau 95$ dimerize through a triple $\beta$-barrel domain consistent with several crosslinks that we observe between their dimerization domains, while the C-terminal DNA-binding domain of $\tau 95$ contains a winged helix domain similar to TFIIF Rap30 (ref. 14). In addition to the $\tau$ IR, our XL-MS data reveal only one other possible link between $\tau \mathrm{A}$ and $\tau \mathrm{B}$ involving the dimerization domain of subunit $\tau 95$ and subunit $\tau 138$. The importance of this link will have to be substantiated in future studies. In $\tau B$, the WD40 propeller subunits $\tau 60$ and $\tau 91$ have been previously proposed to form a platform for $\tau 138$ interaction, thus cooperatively regulating B box binding of $\tau 138$ (refs 12,34). We have observed crosslinks between the $\tau 91$ subunit and a disordered region between the third predicted winged helix domain and the eWH domain of $\tau 138$, which are not detected when TFIIIC is bound to DNA (Supplementary Fig. 1). In addition, it has been shown that a mutation in the third winged helix domain of $\tau 138$ (G349E) strongly reduces the affinity of TFIIIC for DNA ${ }^{18}$. Considering these different bodies of evidence it is tempting to speculate that this region in $\tau 138$ directly recognizes $\mathrm{B}$ box sequences.

In addition to advancing our understanding of TFIIIC architecture, our study also provides surprising insights into the overlap between $\tau \mathrm{A}, \tau \mathrm{B}$ and TFIIIB interaction sites. Previous models have proposed that, due to its intrinsic flexibility, binding sites on the $\tau 131$ TPR array that are crucial for the binding of Brf1 and Bdp1 are masked in the context of the full TPR array and the extended $\mathrm{N}$ terminus ${ }^{27,28}$. This could be an important mode of regulation, preventing the assembly of TFIIIB until conditions are optimal for transcription to proceed. Our results provide new insights into the co-ordination and regulation of this assembly. We find that the extended $\mathrm{N}$ terminus of $\tau 131$ is absolutely required for high-affinity binding to Brf1-TBP. Under our experimental conditions, we were not able to detect an interaction between Brf1-TBP and the TPR array alone, although it seems certain that Brfl can also bind here, as mutations that enhance Brf1 binding and stimulate Pol III transcription have been mapped to TPR 2 on the left arm of the $\operatorname{array}^{26}$ (Fig. 4a). Bdp1 can bind to $\tau 131$ without the presence of Brf1-TBP, and the extended $\mathrm{N}$ terminus of $\tau 131$ plays no role in inhibition of binding. Finally, our competition experiments have demonstrated that $\tau 131$ cannot accommodate $\tau 138$ and Bdp1 at the same time, whereas Brf1-TBP and $\tau 138$ can assemble simultaneously on $\tau 131$. We thus propose that $\tau 138$ prevents complete assembly of TFIIIB, with the crucial Bdp1 binding site masked by a disordered region of $\tau 138$, the $\tau$ IR (Fig. $6 \mathrm{~b}$ ).

On the basis of previous studies and the results presented here, we propose the following sequential model for TFIIIB assemblythe assembly is initiated by the recruitment of Brf1 to $\tau 131$, most likely by the completed assembly of TFIIIC on a tRNA gene. The second TFIIIB component, namely TBP, is then recruited via binding sites on Brfl and via the $\tau \mathrm{B}$ subunit $\tau 60$ (refs 12,37). In the final step of TFIIIB assembly, Bdp1 is recruited to the TFIIIC/ Brf1/TBP complex, competing with $\tau 138$ for a binding hotspot on TPR 8 of $\tau 131$. On binding, Bdp1 causes a conformational change or a break between $\tau A$ and $\tau B$, displacing the critical $\tau$ IR from the TPR array of $\tau 131$ and inducing the displacement of the $\tau B$ module. TFIIIC (with the $\tau \mathrm{B}$ module providing most of the DNA-binding affinity) is only required for assembling TFIIIB but is dispensable for Pol III transcription ${ }^{2}$ and is displaced from its DNA-binding site during Pol III transcription as shown by EMSAs in an in vitro reconstituted system ${ }^{38}$. The mechanism by 
a

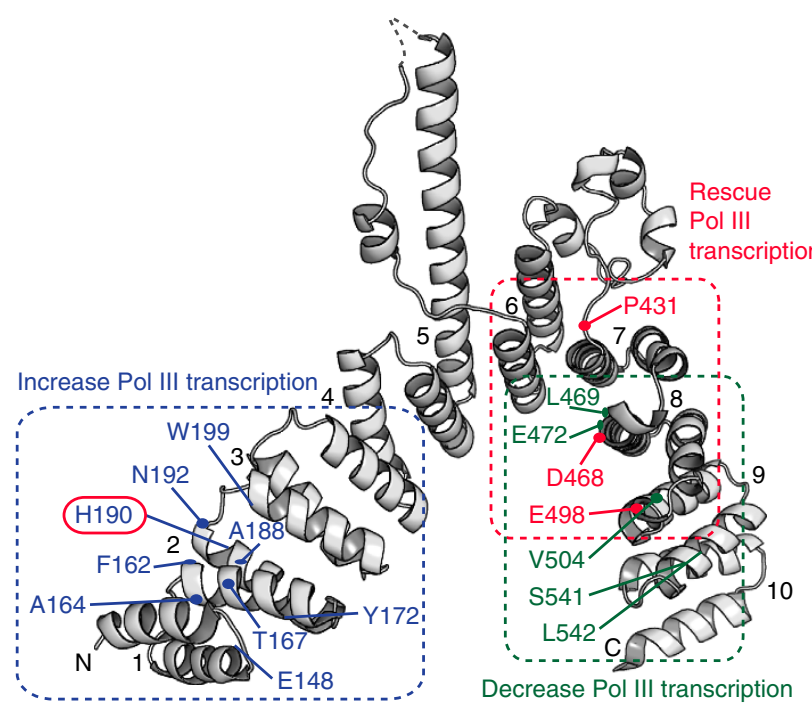

b

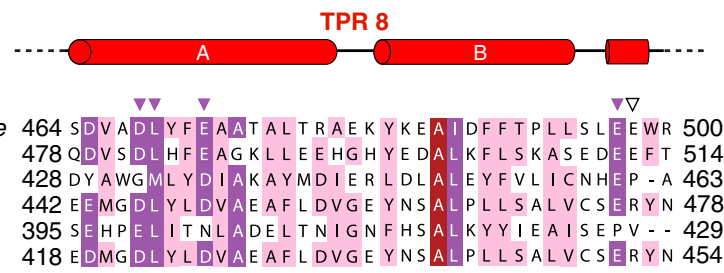

S. cerevisiae 464 SDVADLYFEAATALTRAEK YKEA I DFFTPLLSLEEWR 500 C. albicans 478 QDVSDLHFEAGKLLEEHGHYEDALKFLSKASEDEEFT 514 S. pombe 428 DYAWGMLYDIAKAYMDIERLDLALEYFVL I CNHEP-A 463 G. gallus 442 EEMGDL Y LDVAEAFLDVGE YNSALPLLSALVCSERYN 478 A. thaliana 395 SE HPEL I T NLADELTN I GN FHS AL KYY IEA I SEPV - 429 H. sapiens 418 EDMGDLYLDVAEAFLDVGE YNSALPLLSALVCSERYN 454
C
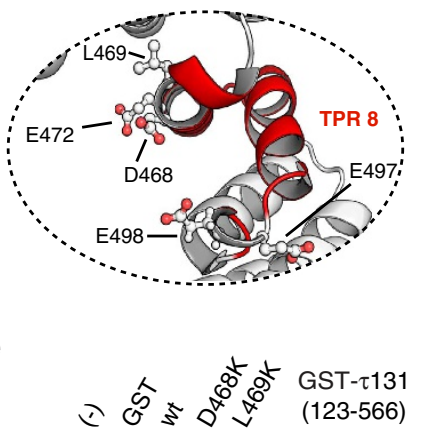

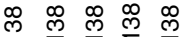

$(\mathrm{eWH}-\tau \mid \mathrm{R})$
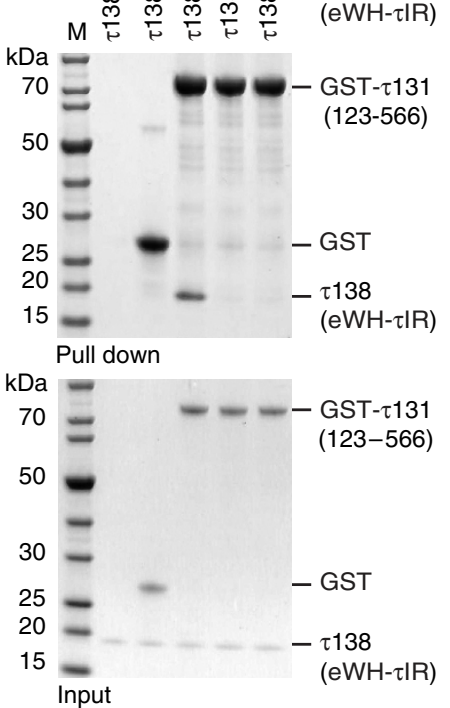

d ITC binding affinities (nM)

\begin{tabular}{|c|c|c|}
\hline $\begin{array}{c}\tau 131 \\
(123-566)\end{array}$ & $\begin{array}{c}\tau 138 \\
(\tau \mathrm{IR})\end{array}$ & $\begin{array}{c}\tau 138 \\
(\mathrm{eWH}-\tau \mathrm{IR})\end{array}$ \\
\hline wt & $80 \pm 14$ & $100 \pm 15$ \\
\hline D468K & No binding & No binding \\
\hline L469K & No binding & No binding \\
\hline E472K & $833 \pm 139$ & $415 \pm 42$ \\
\hline E497K & $89 \pm 19$ & $99 \pm 10$ \\
\hline E498K & $833 \pm 102$ & $971 \pm 217$ \\
\hline
\end{tabular}

f

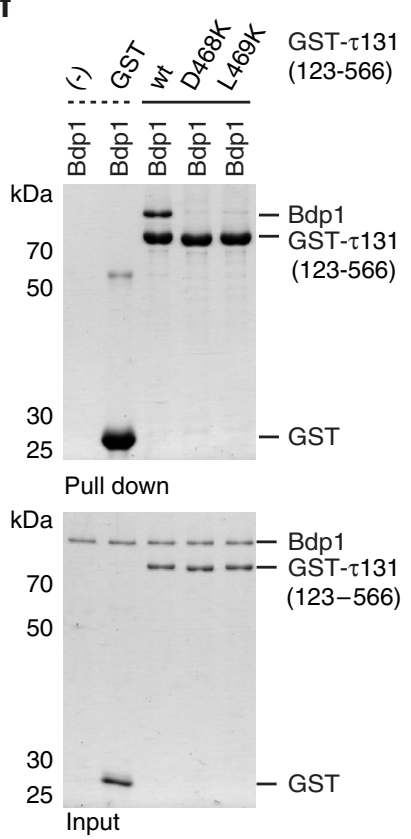

Figure 4 | A binding hotspot on the $\tau \mathbf{1 3 1}$ TPR array for $\tau \mathbf{1 3 8}$ and Bdp1. (a) Mapped mutants of the $\tau 131$ TPR array (see text for details). (b) Sequence alignment of TPR 8. Identical residues are boxed in brick red, highly conserved in purple, medium conserved in pink and low conserved in white. Coloured arrowheads indicate the five mutated residues. (c) Close-up from the structure of TPR 8 . The five residues selected for mutation are displayed as sticks; carbon atoms (grey); oxygen atoms (red). (d) Summary of ITC measurements using indicated $\tau 131$ (123-566) point mutants with $\tau 138$ ( $\tau$ IR) or $\tau 138$ (eWH- $\tau \mid R$ ). Wild-type (wt) measurements are included for reference. (e) GST pull-down assays of purified wild-type (wt) and mutant GST-tagged $\tau 131$ (123-566) variants with untagged $\tau 138$ (eWH- $\tau$ IR). ( - ) indicates a background control for nonspecific binding of $\tau 138$ to the GST-affinity resin. A mixture of purified GST and untagged $\tau 138$ was also used as a negative control. Lower gel shows $5 \%$ of the input and upper gel shows bound fractions. (f) GST pull-down assays of purified wild-type (wt) and mutant GST-tagged $\tau 131$ (123-566) variants with untagged Bdp1. Negative controls and gel format as in $\mathbf{e}$.

which in vivo TFIIIC is displaced (and presumably disassembled) during transcription is currently unknown. However, we suggest that Bdp1 could induce the displacement of the $\tau \mathrm{B}$ module as a regulatory mechanism essential for the initial round of Pol III transcription. Consistent with this model, a recent mass spectrometry study of TAP-purified Pol III from actively transcribing yeast cells detected co-eluting peptides from all three subunits of TFIIIB and peptides from the $\tau 131$ subunit, but not from any $\tau \mathrm{B}$ subunit $^{39}$.

\section{Methods}

Purification of endogenous TFIIIC. TFIIIC was purified from S. cerevisiae strain SC2342 (provided by Cellzome AG), which expresses endogenous TFC8 ( $\tau 60)$ fused with a C-terminal TAP-tag. Yeast cells were grown overnight in YPD medium at $30^{\circ} \mathrm{C}$ and 200 r.p.m. in a BIOSTAT C30 fermenter (Sartorius) under controlled conditions and collected at an $\mathrm{OD}_{600 \mathrm{~nm}}$ of 5-6. The cell paste was resuspended in lysis buffer (250 $\mathrm{mm}$ Tris- $\mathrm{HCl}, \mathrm{pH} 8,40 \%$ glycerol, $250 \mathrm{~mm}$ $\left(\mathrm{NH}_{4}\right)_{2} \mathrm{SO}_{4}, 1 \mathrm{~mm}$ EDTA, $12 \mathrm{~mm} \beta$-mercaptoethanol) supplemented with protease inhibitors (Roche) before being lysed with glass beads in a BeadBeater (BioSpec). The lysate was centrifuged at 14,000 r.p.m. for $1 \mathrm{~h}$ at $4{ }^{\circ} \mathrm{C}$, with the resulting supernatant then loaded onto a Heparin-Sepharose resin (GE Healthcare). The complex was eluted from the resin using high-salt buffer with $1 \mathrm{M}\left(\mathrm{NH}_{4}\right)_{2} \mathrm{SO}_{4}$ and then diluted back to low salt for incubation with IgG Sepharose (GE Healthcare) for $6 \mathrm{~h}$. After washing, IgG beads were incubated with TEV protease overnight at
$4{ }^{\circ} \mathrm{C}$. IgG-cleaved TFIIIC was recovered and subsequently purified by ionic exchange on a MonoQ column (GE Healthcare). TFIIIC was then applied to a Superose $610 / 300$ column which had been pre-equilibrated in the final buffer (25 mm HEPES pH 7.5, $150 \mathrm{~mm} \mathrm{NaCl}, 1 \mathrm{~mm}$ DTT). The eluted protein was subsequently concentrated to $1 \mu \mathrm{g} \mu \mathrm{l}^{-1}$.

Chemical crosslinking of TFIIIC. Thirty $\mu \mathrm{g}\left(1 \mu \mathrm{g} \mu \mathrm{l}^{-1}\right)$ of purified TFIIIC complex was crosslinked by addition of an iso-stoichiometric mixture of H12/D12 isotope-coded, di-succinimidyl-suberate (DSS, Creative Molecules). Equal amounts of crosslinker were added 10 times every $4 \mathrm{~min}$ to a final concentration of $2 \mathrm{~mm}$. The crosslinking reactions were allowed to proceed for $40 \mathrm{~min}$ at $37^{\circ} \mathrm{C}$ and quenched by the addition of ammonium bicarbonate to a final concentration of $50 \mathrm{~mm}$ for $10 \mathrm{~min}$ at $37^{\circ} \mathrm{C}$. Crosslinked proteins were denatured using urea and Rapigest (Waters) at a final concentration of $4 \mathrm{M}$ and $0.05 \%(\mathrm{w} / \mathrm{v})$, respectively. Samples were reduced using $10 \mathrm{mM}$ DTT $\left(30 \mathrm{~min}\right.$ at $\left.37^{\circ} \mathrm{C}\right)$ and cysteines were carbamidomethylated with $15 \mathrm{~mm}$ iodoacetamide (30 min in the dark). Protein digestion was performed first using 1:100 (w/w) LysC (Wako Chemicals, Neuss, Germany) for $3.5 \mathrm{~h}$ at $37^{\circ} \mathrm{C}$ then finalized with $1: 50(\mathrm{w} / \mathrm{w})$ trypsin (Promega, Mannheim, Germany) overnight at $37^{\circ} \mathrm{C}$, after the urea concentration was diluted to $1.5 \mathrm{M}$. Samples were then acidified with $10 \%$ (v/v) TFA and desalted using MicroSpin columns (Harvard Apparatus). Crosslinked peptides were enriched using size-exclusion chromatography (SEC) ${ }^{40}$. In brief, desalted peptides were reconstituted with SEC buffer $(30 \%(\mathrm{v} / \mathrm{v}) \mathrm{ACN}$ in $0.1 \%(\mathrm{v} / \mathrm{v})$ TFA) and fractionated using a Superdex Peptide PC $3.2 / 30$ column (GE) on a Ettan LC system (GE Healthcare) at a flow rate of $50 \mathrm{ml} \mathrm{min}^{-1}$. Fractions eluting between 1 and 
a
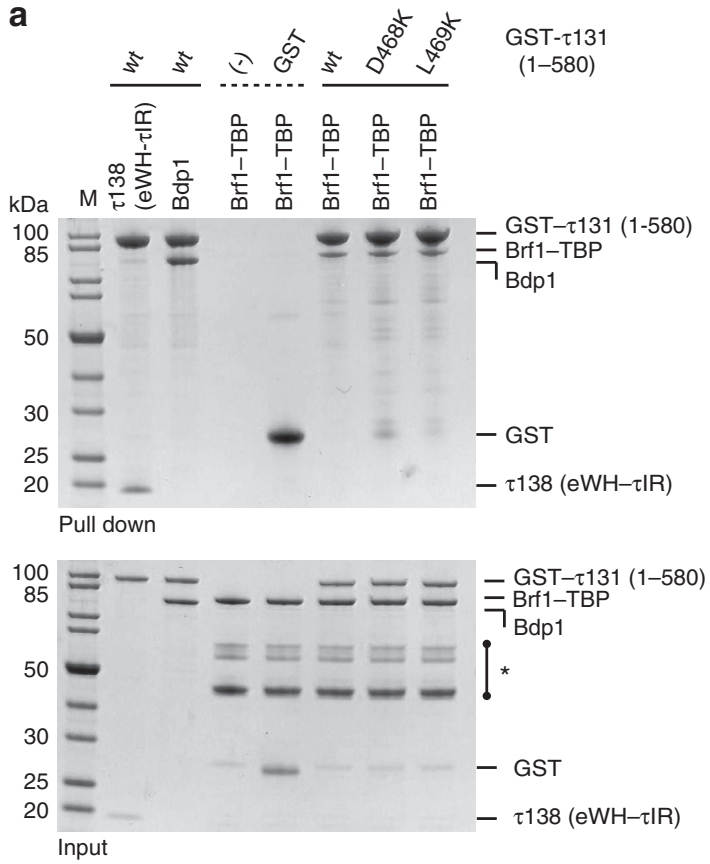
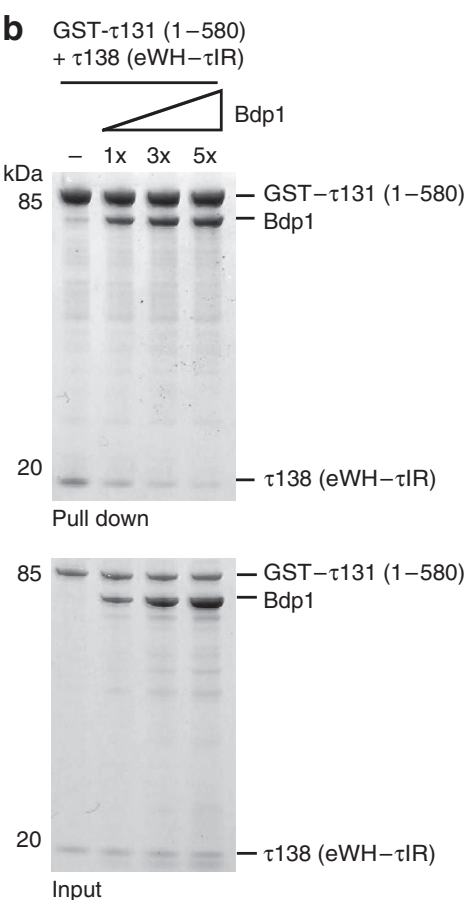
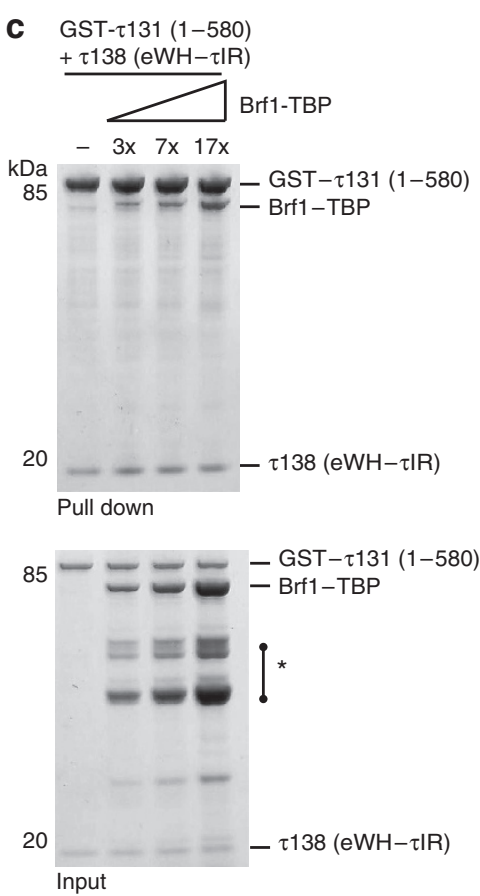

Figure 5 | Defining the overlap between TFIIIB and $\tau 138$ binding to $\tau 131$. (a) GST pull-down assays of purified wild-type (wt) GST-tagged $\tau 131$ (1-580) with untagged $\tau 138$ (eWH- $\tau \mid \mathrm{R}$ ) and Bdp1, and purified wild-type (wt) and mutant GST-tagged $\tau 131$ (1-580) variants with Brf1-TBP. ( - ) indicates a background control for nonspecific binding of Brf1-TBP to the GST-affinity resin. A mixture of purified GST and untagged Brf1-TBP was also used as a negative control. Lower gel shows $5 \%$ of the input and upper gel shows bound fractions. An * in the input gel indicates degradation products of Brf1-TBP. (b) GST pull-down competition assays of purified wild-type (wt) GST-tagged $\tau 131$ (1-580) with untagged $\tau 138$ (eWH- $\tau$ IR) and Bdp1. GST- $\tau 131$ was preincubated with $\tau 138$ before addition of the indicated molar excess of Bdp1. ( - ) indicates a control experiment where no Bdp1 was added. Gel format as in a. (c) GST pull-down competition assays of purified wild-type (wt) GST-tagged $\tau 131$ (1-580) with untagged $\tau 138$ (eWH- $\tau$ IR) and Brf1-TBP. GST- $\tau 131$ was preincubated with $\tau 138$ before addition of the indicated molar excess of Brf1-TBP. $(-)$ indicates a control experiment where no Brf1-TBP was added. Gel format as in $\mathbf{a}$. An * in the input gel indicates degradation products of Brf1-TBP.

$1.5 \mathrm{ml}$ were evaporated to dryness and reconstituted in $50 \mu \mathrm{l} 5 \%(\mathrm{v} / \mathrm{v}) \mathrm{ACN}$ in $0.1 \%(\mathrm{v} / \mathrm{v}) \mathrm{FA}$.

Mass spectrometry analysis of crosslinked peptides. Between $2 \%$ and $10 \%$ of the amount contained in the collected SEC fractions were analysed by liquid chromatography-coupled tandem mass spectrometry (MS/MS) using a nanoAcquity UPLC system (Waters) connected online to LTQ-Orbitrap Velos Pro instrument (Thermo). Peptides were separated on a BEH300 C18 $(75 \mathrm{~mm} \times 250$ $\mathrm{mm}, 1.7 \mathrm{~mm}$ ) nanoAcquity UPLC column (Waters) using a stepwise $60 \mathrm{~min}$ gradient between $3 \%$ and $85 \%(\mathrm{v} / \mathrm{v}) \mathrm{ACN}$ in $0.1 \%(\mathrm{v} / \mathrm{v})$ FA. Data acquisition was performed using a TOP-20 strategy where survey-MS scans $(\mathrm{m} / \mathrm{z}$ range $375-1,600)$ were acquired in the Orbitrap $(R=30,000)$ and up to 20 of the most abundant ions per full scan were fragmented by collision-induced dissociation (normalized collision energy $=40$, activation $Q=0.250$ ) and analysed in the $L T Q$. To focus the acquisition on larger crosslinked peptides, charge states 1,2 and unknown were rejected. Dynamic exclusion was enabled with repeat count $=1$, exclusion duration $=60 \mathrm{~s}$, list size $=500$ and mass window \pm 15 p.p.m. Ion target values were $1,000,000$ (or $500 \mathrm{~ms}$ maximum fill time) for full scans and 10,000 (or $50 \mathrm{~ms}$ maximum fill time) for MS/MS scans. All the samples were analysed in technical duplicates. To assign the fragment ion spectra, raw files were converted to centroid mzXML using the Mass Matrix file converter tool and then searched using $\mathrm{xQuest}^{41}$ against a fasta database containing the sequences of the crosslinked proteins. Posterior probabilities were calculated using xProphet ${ }^{41}$ and results were filtered using the following parameters: false discovery rate $=0.05$, min delta score $=0.95$, MS1 tolerance window of 4 to 7 p.p.m., ld-score $>25$.

Yeast strains. Plasmid pOL49 (a kind gift from O. Lefebvre) carrying a wild-type copy of $\tau 138$ (ref. 42) was transformed into a diploid yeast strain carrying a chromosomal deletion of $\tau 138$ (Euroscarf, Acc. Number Y20406). Haploid segregants carrying the $\tau 138$ deletion and the pOL49 plasmid were identified and isolated. $\tau 138$ was cloned by PCR amplification of genomic DNA, with primers spanning $500 \mathrm{bp}$ up- and downstream of the coding sequence, and inserted into a plasmid carrying LEU selection (pRS415) to obtain pRS415- $\tau 138$. pRS415 is originally a CEN plasmid but since the genomic copy of $\tau 138$ carries a CEN sequence, this was removed from the pRS415- $\tau 138$ plasmid via restriction-free cloning ${ }^{43}$. The new plasmid was named pRS415 $\Delta$ CEN $\tau 138$. Restriction-free cloning was performed on plasmid pRS415 $\Delta$ CEN $\tau 138$ to create the deletion mutants.

Cell viability-spot assays. Yeast strain carrying the plasmid pOL49 was transformed with the pRS415 $\Delta$ CEN $\tau 138$ plasmid set (including wild type, mutants and empty plasmid without $\tau 138$ gene) and plated on selective media (to select for both plasmids). Single colonies from fresh plates grown for 2 days at $30^{\circ} \mathrm{C}$ were suspended in PBS to a final $\mathrm{OD}_{600 \mathrm{~nm}}$ of 0.4 . Serial 10 -fold dilutions of the different transformants were spotted on SC-LEU medium and 5-fluoroorotic acid containing medium, and incubated for $72 \mathrm{~h}$ at $30^{\circ} \mathrm{C} . n=3$.

Protein expression and purification. The sequences for $\tau 131$ (123-566) and fulllength Bdp1 were cloned into the pETM11 vector in frame with a TEV proteasecleavable N-terminal 6xHis tag and were transformed into BL21 Star (DE3) pRARE E. coli cells for expression. The codon-optimized sequence for $\tau 138$ (546-641) was cloned into the pETM 30 vector in frame with a TEV protease-cleavable $\mathrm{N}$-terminal 6xHis-GST tag and was transformed into BL21 (DE3) Gold E. coli cells for expression.

Cells were grown in $\mathrm{TB}$ till an $\mathrm{OD}_{600 \mathrm{~nm}}$ of 0.8 , and protein expression was induced for $16 \mathrm{~h}$ at $18^{\circ} \mathrm{C}$ with $0.5 \mathrm{mM}$ isopropyl- $\beta$-D-thiogalactoside. Cells were harvested by centrifugation and resuspended in buffer A (5\% glycerol, $50 \mathrm{~mm}$ Tris $\mathrm{pH} 7.5,500 \mathrm{mM} \mathrm{NaCl}, 20 \mathrm{mM}$ imidazole, $4 \mathrm{mM} \mathrm{MgCl}_{2}$, Complete EDTA-free Protease Inhibitor Cocktail (Roche), DNasel (Roche), $2 \mathrm{mM} \beta$-mercaptoethanol) before being lysed by homogenization (Avestin Emulsiflex-C3). The lysate was centrifuged at 20,000 r.p.m. and the resulting supernatant was incubated with $2 \mathrm{ml}$ Nickel-NTA agarose resin (Qiagen), preincubated in buffer B (50 mM Tris pH 7.5, $300 \mathrm{mM} \mathrm{NaCl}, 20 \mathrm{mM}$ imidazole, $2 \mathrm{mM} \beta$-mercaptoethanol), for $1 \mathrm{~h}$ at $4{ }^{\circ} \mathrm{C}$. The resin was applied to a disposable column and washed with 20 column volumes of buffer $\mathrm{B}, 20$ column volumes of buffer $\mathrm{B}$ containing $1 \mathrm{M} \mathrm{NaCl}$ and again with 20 column volumes of buffer B. The protein was eluted off the column using buffer $B$ containing $250 \mathrm{mM}$ imidazole and incubated with TEV protease for $16 \mathrm{~h}$ at $4{ }^{\circ} \mathrm{C}$ in buffer B. Cleaved protein was reapplied to the resin and collected in the flowthrough, before being applied to a pre-equilibrated $(20 \mathrm{mM}$ Tris pH 7.5, $150 \mathrm{mM}$ $\mathrm{NaCl}, 2 \mathrm{mM}$ DTT) preparative S200 26/60 column (GE Healthcare). Cloning of the 


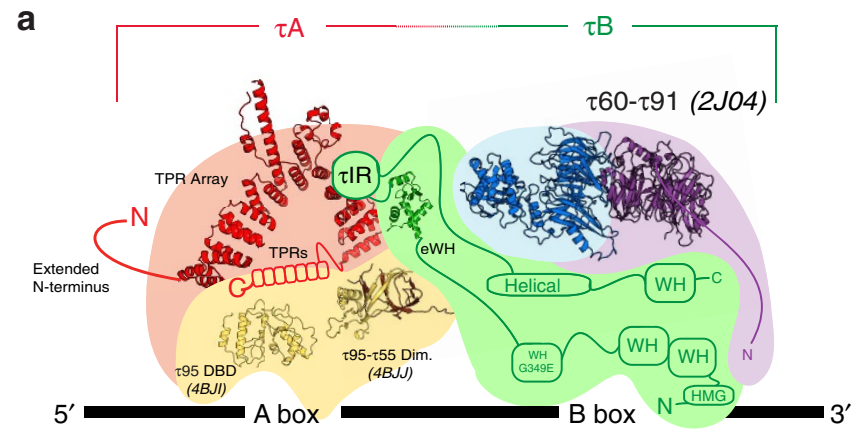

b

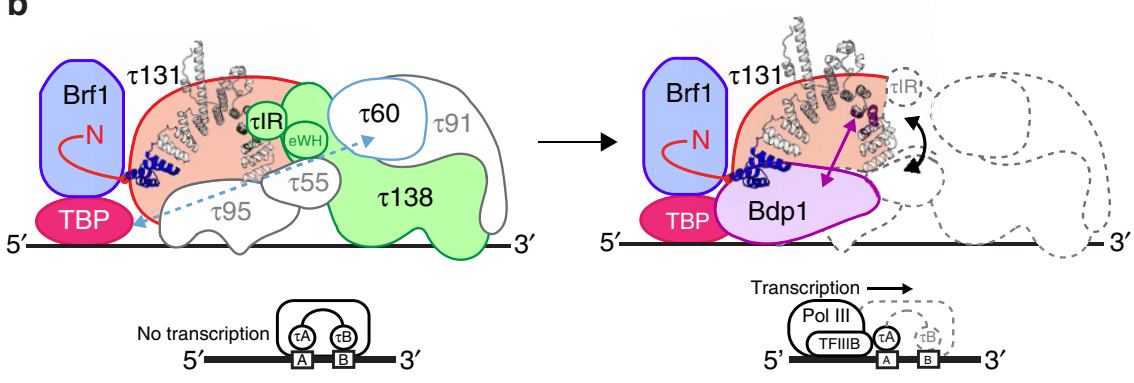

Figure 6 | The role of $\tau \mathbf{1 3 1}$ in Pol III PIC formation. (a) Our current view of the arrangement of $\tau A$ and $\tau B$ subunits within TFIIIC based on interaction studies, crystal structures and crosslinking. Structures from this study are included, as well as previous structures with PDB codes indicated: the WD40 dimer structure of $\tau 60-\tau 91$ (ref. 12), and the $\tau 95$ DNA-binding domain (DBD) ${ }^{14}$ and $\tau 95-\tau 55$ dimerization homologues from S. pombe ${ }^{14}$. Note that the non-conserved $\tau 55$ histidine phosphatase domain (HPD) is omitted ${ }^{13}$. The extended $N$ terminus and C-terminal TPRs of $\tau 131$ are indicated schematically. Predicted structural regions of $\tau 138$ are also indicated, including the G349E mutation. The disordered N terminus of $\tau 91$ is included schematically.

(b) Model indicating two stages of PIC formation. In the first stage, recruitment of Brf1 to the PIC requires the extended N terminus of $\tau 131$ (red curve) and the N-terminal TPRs of the TPR array (highlighted in blue). TBP makes interactions with Brf1 and the $\tau 60$ subunit of $\tau$ B. The link between $\tau 131$ and $\tau 138$ is maintained. In the second stage, the recruitment of $B d p 1$ involves conformational changes in the arms of the TPR array. $\tau I R$ is displaced and the $\tau A-\tau B$ link is altered, possibly leading to the disassembly of TFIIIC. TFIIIB is now assembled and recruits Pol III, together with $\tau 131$, for transcription.

$\tau 131$ point mutants was carried out using protocols and reagents provided in the Quikchange Lightning kit (Agilent). Mutants were purified by the same method as the wild type.

Protein expression and purification of Brf1-TBP. The sequence of the Brf1-TBP fusion protein Brf1 (1-382)-TBP (61-240)-Brf1 (439-596) has been described previously ${ }^{36}$. The sequence was cloned into the pETM13 vector in frame with a non-cleavable C-terminal 6xHis tag. The vector was transformed into BL21 Star (DE3) pRARE E. coli cells and grown in TB till an $\mathrm{OD}_{600 \mathrm{~nm}}$ of 0.8 . Protein expression was induced for $16 \mathrm{~h}$ at $18^{\circ} \mathrm{C}$ with $0.5 \mathrm{~mm}$ isopropyl- $\beta$-D-thiogalactoside. Lysis and histidine-affinity purification was as described above, but with $5 \%$ glycerol added to all 'B' buffers. Eluted protein was diluted in buffer C ( $5 \%$ glycerol, $50 \mathrm{mM}$ Tris $\mathrm{pH} 7.5,2 \mathrm{mM}$ DTT) so that the final $\mathrm{NaCl}$ concentration was $150 \mathrm{mM}$. The sample was applied to a $5 \mathrm{ml}$ HiTrap SP HP column (GE Healthcare) that had been pre-equilibrated in buffer D (5\% glycerol, $50 \mathrm{mM}$ Tris pH 7.5, $150 \mathrm{mM} \mathrm{NaCl}$, $2 \mathrm{mM}$ DTT). The column was washed with 20 column volumes of buffer D, before a gradient of 40 column volumes into buffer E (5\% glycerol, $50 \mathrm{~mm}$ Tris pH 7.5, $1 \mathrm{M} \mathrm{NaCl}, 2 \mathrm{mM}$ DTT) was applied.

Crystallization and X-ray structure determination. For $\tau 131$ (123-566), crystals of the $\mathrm{P}_{3}$ and $\mathrm{P}_{2}$ space group were grown at $20^{\circ} \mathrm{C}$ by the hanging-drop vapour diffusion method at a concentration of $49 \mathrm{mg} \mathrm{ml}^{-1}$ and $60 \mathrm{mg} \mathrm{ml}^{-1}$ respectively, with a 1:1 ratio of protein and crystallization solutions. For $\mathrm{P}_{3}$, the crystallization solution contained $0.1 \mathrm{M}$ bicine $\mathrm{pH} 8.9$ and $0.85 \mathrm{M} \mathrm{MgCl}_{2}$. Crystals were cryo-protected by soaking in mother liquor containing $30 \%$ glycerol before being flash-frozen in liquid nitrogen. For $\mathrm{P}_{2}$, the crystallization solution contained $0.2 \mathrm{M}$ $\mathrm{MgCl}_{2}, 0.1 \mathrm{M}$ Tris pH 8.3 and $42.5 \%$ ethylene glycol. Crystals were flash-frozen in liquid nitrogen using the already present ethylene glycol as a cryo-protectant. X-ray data for native, selenomethionine-incorporated and $\mathrm{Hg}$ crystals were collected at the ESRF and PETRAIII beamlines. The data was processed with X-ray diffuse scattering (XDS ${ }^{44}$. The $\mathrm{P}_{3}$ crystals were also consistently indexed with pointless and each data set was further scaled with SCALA. Derivatives were scaled to the native data set with scaleit. The $\mathrm{P} 6_{2}$ structure was solved by SAD combined with density modification using the programme autoSHARP ${ }^{45}$. The $\mathrm{P}_{3}$ structure was solved by MIRAS combined with density modification using autoSHARP. For both structures, iterative model building and refinement was carried out using $\operatorname{Coot}^{46}$ and Phenix ${ }^{47}$, respectively. The initial models were used as molecular replacements models for native, higher-resolution data sets using PHASER ${ }^{48}$. The final models were validated using MolProbity ${ }^{49}$.

For $\tau 138$ (546-641), crystals were grown at $20^{\circ} \mathrm{C}$ by the hanging-drop vapour diffusion method at a concentration of $30 \mathrm{mg} \mathrm{ml}^{-1}$. Protein solution and crystallization solution were mixed in a 1:1 ratio. The crystallization solution contained $1.15 \mathrm{M} \mathrm{Na}$ citrate $\mathrm{pH} 6.2$ and $0.1 \mathrm{M} \mathrm{Na}$ cacodylate. Crystals were cryo-protected by soaking in mother liquor containing $15 \%$ glycerol before being flash-frozen in liquid nitrogen. Data were collected on an in-house rotating anode to record single-wavelength anomalous signal from sulphur atoms. The data were processed using XDS. The initial substructure, based on anomalous signal from sulphur atoms, was solved using autoSHARP. Six sulphur sites were identified, indicating two molecules in the asymmetric unit. Phasing equations were solved and density modification was performed using autoSharp. Automatic building of an initial model was carried out using AutoBuild in Phenix. A higher-resolution native data set on a second crystal was collected at the ID23-1 beamline at the ESRF, and was processed using XDS. The structure was solved by molecular replacement with Phaser using the initial model from the sulphur-SAD experiment above. Iterative model building and refinement was then carried out using Coot and Phenix respectively. The final model was validated using MolProbity.

GST pull-down experiments. GST-tagged $\tau 131$ and $\tau 138$ proteins were expressed in BL21 (DE3) Gold E. coli cells, and purified with a GST preparative FF 16/10 column (GE Healthcare) followed by size-exclusion chromatography using a pre-equilibrated (20 mm Tris pH 7.5, $150 \mathrm{mM} \mathrm{NaCl}, 2 \mathrm{mM}$ DTT) S200 26/60 column (GE Healthcare). For pull-down experiments, $15 \mu \mathrm{g}$ of GST-tagged $\tau 131$ or $\tau 138$ protein and their tested binding partners were incubated with $25 \mu \mathrm{l}$ Glutathione Sepharose 4B beads in buffer F (50 mM Tris pH 7.5, $150 \mathrm{mM} \mathrm{NaCl}$, $2 \mathrm{mM}$ DTT, $0.1 \%$ Tween 20$)$ at $4{ }^{\circ} \mathrm{C}$ for $4 \mathrm{~h} . \tau 138(300 \mathrm{nM})$ and Bdp1, and $1 \mu \mathrm{M}$ of Brf1-TBP were used when testing binding to the GST- $\tau 131$ constructs. Wild-type (900 nM) and mutant $\tau 131$ was used when testing the binding to GST- $\tau 138$ (641-693). After incubation, the beads were washed three times with buffer F, before being heated to $100^{\circ} \mathrm{C}$ in Laemmli sample buffer for $5 \mathrm{~min}$.

ITC experiments. ITC was performed using a MicroCal ITC200 System (GE Healthcare). All samples were dialyzed into ITC buffer $(20 \mathrm{mM}$ Tris $\mathrm{pH} 7.5$, 
$150 \mathrm{mM} \mathrm{NaCl}, 2 \mathrm{mM} \beta$-mercaptoethanol). Protein concentration in the cell and syringe was 15 and $150 \mu \mathrm{M}$, respectively. Experiments were performed at $25^{\circ} \mathrm{C}$.

EMSA of TFIIIC and $\tau 138$ (546-641). The sequence of the 66 base-pair oligonucleotide (5'-CGA TAT AGT GTA ACG GCT ATC ACA TCA CGC TTT CAC CGT GGA GAC CGG GGT TCG ACT CCC CGT ATC-3') contains A and B box elements from a tDNA Glu sequence (underlined). HPLC-purified oligonucleotides were ${ }^{32} \mathrm{P}$ end-labelled by T4 Polynucleotide Kinase before subsequent gel purification by denaturing $15 \%$ urea-PAGE. To form double-stranded oligonucleotides, the ${ }^{32} \mathrm{P}$-labelled oligonucleotides were heated to $95^{\circ} \mathrm{C}$ for $2 \mathrm{~min}$, cooled to $25^{\circ} \mathrm{C}$ and incubated in EMSA buffer $(20 \mathrm{mM}$ Tris $\mathrm{pH} 7.5,150 \mathrm{mM} \mathrm{NaCl}$, $1 \mathrm{mM} \mathrm{MgCl} 2,2 \mathrm{mM}$ DTT). For the EMSA, single-stranded or double-stranded oligonucleotides were incubated with TFIIIC or $\tau 138(546-641)$ at $25^{\circ} \mathrm{C}$ for $30 \mathrm{~min}$, before being run at $120 \mathrm{~V}$ on a $4.5 \%$ acrylamide gel in Tris-glycine buffer. The gel was dried and autoradiographed with X-ray film (Biomax, MR-film, Kodak).

\section{References}

1. Schramm, L. \& Hernandez, N. Recruitment of RNA polymerase III to its target promoters. Genes Dev. 16, 2593-2620 (2002).

2. Kassavetis, G. A., Braun, B. R., Nguyen, L. H. \& Geiduschek, E. P. S. cerevisiae TFIIIB is the transcription initiation factor proper of RNA polymerase III, while TFIIIA and TFIIIC are assembly factors. Cell 60, 235-245 (1990).

3. Geiduschek, E. P. \& Kassavetis, G. A. The RNA polymerase III transcription apparatus. J. Mol. Biol. 310, 1-26 (2001).

4. Conesa, C., Swanson, R. N., Schultz, P., Oudet, P. \& Sentenac, A. On the subunit composition, stoichiometry, and phosphorylation of the yeast transcription factor TFIIIC/tau. J. Biol. Chem. 268, 18047-18052 (1993).

5. Ducrot, C. et al. Reconstitution of the yeast RNA polymerase III transcription system with all recombinant factors. J. Biol. Chem. 281, 11685-11692 (2006).

6. Stillman, D. J. \& Geiduschek, E. P. Differential binding of a S. cerevisiae RNA polymerase III transcription factor to two promoter segments of a tRNA gene. EMBO J. 3, 847-853 (1984).

7. Baker, R. E., Camier, S., Sentenac, A. \& Hall, B. D. Gene size differentially affects the binding of yeast transcription factor tau to two intragenic regions. Proc. Natl Acad. Sci. USA 84, 8768-8772 (1987).

8. Nagarajavel, V., Iben, J. R., Howard, B. H., Maraia, R. J. \& Clark, D. J. Global 'bootprinting' reveals the elastic architecture of the yeast TFIIIB-TFIIIC transcription complex in vivo. Nucleic Acids Res. 41, 8135-8143 (2013).

9. Marzouki, N., Camier, S., Ruet, A., Moenne, A. \& Sentenac, A. Selective proteolysis defines two DNA binding domains in yeast transcription factor tau. Nature 323, 176-178 (1986).

10. Schultz, P. et al. The two DNA-binding domains of yeast transcription factor tau as observed by scanning transmission electron microscopy. EMBO J. 8, 3815-3824 (1989)

11. Dumay-Odelot, H. et al. Identification, molecular cloning, and characterization of the sixth subunit of human transcription factor TFIIIC. J. Biol. Chem. 282, 17179-17189 (2007).

12. Mylona, A. et al. Structure of the tau60/Delta tau91 subcomplex of yeast transcription factor IIIC: insights into preinitiation complex assembly. Mol. Cell 24, 221-232 (2006)

13. Taylor, N. M. et al. Structural and functional characterization of a phosphatase domain within yeast general transcription factor IIIC. J. Biol. Chem. 288, 15110-15120 (2013)

14. Taylor, N. M., Baudin, F., von Scheven, G. \& Muller, C. W. RNA polymerase III-specific general transcription factor IIIC contains a heterodimer resembling TFIIF Rap30/Rap74. Nucleic Acids Res. 41, 9183-9196 (2013).

15. Jourdain, S., Acker, J., Ducrot, C., Sentenac, A. \& Lefebvre, O. The tau95 subunit of yeast TFIIIC influences upstream and downstream functions of TFIIIC.DNA complexes. J. Biol. Chem. 278, 10450-10457 (2003).

16. Rozenfeld, S. \& Thuriaux, P. Genetic interactions within TFIIIC, the promoterbinding factor of yeast RNA polymerase III. Mol. Genet. Genomics 265, 705-710 (2001).

17. Bartholomew, B., Kassavetis, G. A., Braun, B. R. \& Geiduschek, E. P. The subunit structure of Saccharomyces cerevisiae transcription factor IIIC probed with a novel photocrosslinking reagent. EMBO J. 9, 2197-2205 (1990).

18. Lefebvre, O., Ruth, J. \& Sentenac, A. A mutation in the largest subunit of yeast TFIIIC affects tRNA and 5S RNA synthesis. Identification of two classes of suppressors. J. Biol. Chem. 269, 23374-23381 (1994).

19. Dumay-Odelot, H., Acker, J., Arrebola, R., Sentenac, A. \& Marck, C. Multiple roles of the tau131 subunit of yeast transcription factor IIIC (TFIIIC) in TFIIIB assembly. Mol. Cell. Biol. 22, 298-308 (2002).

20. Zeytuni, N. \& Zarivach, R. Structural and functional discussion of the tetra-trico-peptide repeat, a protein interaction module. Structure 20, 397-405 (2012).

21. Halbach, F., Reichelt, P., Rode, M. \& Conti, E. The yeast ski complex: crystal structure and RNA channeling to the exosome complex. Cell 154, 814-826 (2013).
22. Liao, Y., Willis, I. M. \& Moir, R. D. The Brf1 and Bdp1 subunits of transcription factor TFIIIB bind to overlapping sites in the tetratricopeptide repeats of $\mathrm{Tfc} 4$. J. Biol. Chem. 278, 44467-44474 (2003).

23. Moir, R. D., Puglia, K. V. \& Willis, I. M. A gain-of-function mutation in the second tetratricopeptide repeat of TFIIIC131 relieves autoinhibition of Brf1 binding. Mol. Cell. Biol. 22, 6131-6141 (2002).

24. Moir, R. D., Puglia, K. V. \& Willis, I. M. Autoinhibition of TFIIIB70 binding by the tetratricopeptide repeat-containing subunit of TFIIIC. J. Biol. Chem. 277, 694-701 (2002).

25. Moir, R. D., Puglia, K. V. \& Willis, I. M. Interactions between the tetratricopeptide repeat-containing transcription factor TFIIIC131 and its ligand, TFIIIB70. Evidence for a conformational change in the complex. J. Biol. Chem. 275, 26591-26598 (2000).

26. Moir, R. D., Sethy-Coraci, I., Puglia, K., Librizzi, M. D. \& Willis, I. M. A tetratricopeptide repeat mutation in yeast transcription factor IIIC131 (TFIIIC131) facilitates recruitment of TFIIB-related factor TFIIIB70. Mol. Cell. Biol. 17, 7119-7125 (1997).

27. Liao, Y., Moir, R. D. \& Willis, I. M. Interactions of Brf1 peptides with the tetratricopeptide repeat-containing subunit of TFIIIC inhibit and promote preinitiation complex assembly. Mol. Cell. Biol. 26, 5946-5956 (2006).

28. Moir, R. D. \& Willis, I. M. Tetratricopeptide repeats of Tfc4 and a limiting step in the assembly of the initiation factor TFIIIB. Adv. Protein Chem. 67, 93-121 (2004).

29. Kassavetis, G. A. et al. The role of the TATA-binding protein in the assembly and function of the multisubunit yeast RNA polymerase III transcription factor, TFIIIB. Cell 71, 1055-1064 (1992).

30. Wu, C. C., Lin, Y. C. \& Chen, H. T. The TFIIF-like Rpc37/53 dimer lies at the center of a protein network to connect TFIIIC, Bdp1, and the RNA polymerase III active center. Mol. Cell. Biol. 31, 2715-2728 (2011).

31. Dumay, H., Rubbi, L., Sentenac, A. \& Marck, C. Interaction between yeast RNA polymerase III and transcription factor TFIIIC via ABC10alpha and tau131 subunits. J. Biol. Chem. 274, 33462-33468 (1999).

32. Hsieh, Y. J., Wang, Z., Kovelman, R. \& Roeder, R. G. Cloning and characterization of two evolutionarily conserved subunits (TFIIIC102 and TFIIIC63) of human TFIIIC and their involvement in functional interactions with TFIIIB and RNA polymerase III. Mol. Cell. Biol. 19, 4944-4952 (1999).

33. Kosinski, J. et al. Xlink Analyzer: Software for analysis and visualization of cross-linking data in the context of three-dimensional structures. J. Struct. Biol. (2015).

34. Arrebola, R. et al. Tau91, an essential subunit of yeast transcription factor IIIC, cooperates with tau138 in DNA binding. Mol. Cell. Biol. 18, 1-9 (1998).

35. Meinhart, A., Blobel, J. \& Cramer, P. An extended winged helix domain in general transcription factor E/IIE alpha. J. Biol. Chem. 278, 48267-48274 (2003).

36. Kassavetis, G. A., Soragni, E., Driscoll, R. \& Geiduschek, E. P. Reconfiguring the connectivity of a multiprotein complex: fusions of yeast TATA-binding protein with Brfl, and the function of transcription factor IIIB. Proc. Natl Acad. Sci. USA 102, 15406-15411 (2005).

37. Deprez, E., Arrebola, R., Conesa, C. \& Sentenac, A. A subunit of yeast TFIIIC participates in the recruitment of TATA-binding protein. Mol. Cell. Biol. 19, 8042-8051 (1999).

38. Bardeleben, C., Kassavetis, G. A. \& Geiduschek, E. P. Encounters of Saccharomyces cerevisiae RNA polymerase III with its transcription factors during RNA chain elongation. J. Mol. Biol. 235, 1193-1205 (1994).

39. Nguyen, N. T., Saguez, C., Conesa, C., Lefebvre, O. \& Acker, J. Identification of proteins associated with RNA polymerase III using a modified tandem chromatin affinity purification. Gene 556, 51-60 (2015).

40. Leitner, A. et al. Expanding the chemical cross-linking toolbox by the use of multiple proteases and enrichment by size exclusion chromatography. Mol. Cell. Proteomics 11, 014126 (2012).

41. Walzthoeni, T. et al. False discovery rate estimation for cross-linked peptides identified by mass spectrometry. Nat. Methods 9 , 901-903 (2012).

42. Lefebvre, O. et al. TFC3: gene encoding the B-block binding subunit of the yeast transcription factor IIIC. Proc. Natl Acad. Sci. USA 89, 10512-10516 (1992).

43. Boeke, J. D., LaCroute, F. \& Fink, G. R. A positive selection for mutants lacking orotidine-5'-phosphate decarboxylase activity in yeast: 5 -fluoro-orotic acid resistance. Mol. Gen. Genet. 197, 345-346 (1984).

44. Kabsch, W. Xds. Acta Crystallogr. D Biol. Crystallogr. 66, 125-132 (2010)

45. Vonrhein, C., Blanc, E., Roversi, P. \& Bricogne, G. Automated structure solution with autoSHARP. Methods Mol. Biol. 364, 215-230 (2007).

46. Emsley, P., Lohkamp, B., Scott, W. G. \& Cowtan, K. Features and development of Coot. Acta Crystallogr. D Biol. Crystallogr. 66, 486-501 (2010).

47. Adams, P. D. et al. PHENIX: a comprehensive Python-based system for macromolecular structure solution. Acta Crystallogr. D Biol. Crystallogr. 66, 213-221 (2010).

48. McCoy, A. J. et al. Phaser crystallographic software. J. Appl. Crystallogr. 40, 658-674 (2007). 
49. Chen, V. B. et al. MolProbity: all-atom structure validation for macromolecular crystallography. Acta Crystallogr. D Biol. Crystallogr. 66, 12-21 (2010).

50. Combe, C. W., Fischer, L. \& Rappsilber, J. xiNET: cross-link network maps with residue resolution. Mol. Cell. Proteomics 14, 1137-1147 (2015).

51. Karplus, P. A. \& Diederichs, K. Linking crystallographic model and data quality. Science 336, 1030-1033 (2012).

\section{Acknowledgements}

We acknowledge support by the EMBL Proteomics Core Facility, the EMBL Heidelberg Crystallization Platform and access and support at the ESRF beamlines by the EMBL-ESRF Joint Structural Biology Group and at PETRAIII beamlines. We also thank O. Lefebvre for yeast plasmids and crucial advice; V. Rybin for carrying out ITC experiments, N. Hoffman for support in the recombinant expression of TFIIIB subunits, J. Kosinski for bioinformatics support and I. Kucinski and F. Rodriguez-Calviño for their work during the early stages of the project. C.W.M. acknowledges support by an Advanced Grant of the European Research Council (ERC-2013-AdG340964-POL1PIC). M.C. acknowledges support by the Marie-Curie fellowship (FP7-PEOPLE-2012-IEF 329322). All authors acknowledge support by EMBL.

\section{Author contributions}

G.M., N.M.I.T. and S.G. determined and analysed the different crystal structures; G.M., N.M.I.T. and H.G. carried out the biochemical analysis; M.C. and H.G. produced the yeast strains, A.v.A. and M.B. carried out and analysed the crosslinking experiments; G.M., S.G., M.B. and C.W.M. wrote the manuscript with input from all authors.

\section{Additional information}

Accession codes: The coordinates and structure factors of the $\tau 131$ TPR array in space group P43 and P62 (PDB 5AEM and 5AIO) and $\tau 138$ eWH (PDB 5AIM) have been deposited with the European Protein Data Bank.

Supplementary Information accompanies this paper at http://www.nature.com/ naturecommunications

Competing financial interests: The authors declare no competing financial interests.

Reprints and permission information is available online at http://npg.nature.com/ reprintsandpermissions/

How to cite this article: Male, G. et al. Architecture of TFIIIC and its role in RNA polymerase III pre-initiation complex assembly. Nat. Commun. 6:7387 doi: $10.1038 /$ ncomms8387 (2015).

\section{(c) (i)}

This work is licensed under a Creative Commons Attribution 4.0 International License. The images or other third party material in this article are included in the article's Creative Commons license, unless indicated otherwise in the credit line; if the material is not included under the Creative Commons license, users will need to obtain permission from the license holder to reproduce the material. To view a copy of this license, visit http://creativecommons.org/licenses/by/4.0/ 